\section{BASIC-PLUS programs to manage a small primate colony information and records system}

\author{
RICHARD DENI, JOSEPH ERWIN, \\ DANIEL I. DRAKE, and ELLICE KASSORLA \\ Comparative Development Laboratory, \\ Peabody College, Box 154, \\ Nashville, Tennessee 37203
}

Small nonhuman primate breeding colonies are maintained in a variety of settings including drug companies, research institutes, university departments, and hospitals (National Research Council on Conservation of Nonhuman Primates, 1975). Accurate and accessible records are vital to many of the biomedical and behavioral research plans now under way, and a computer-based information system can meet or exceed many of the record-keeping needs of these small colony facilities.

The BASIC-PLUS programs (Digital Equipment Corporation, 1975) described here are designed to set up and manage an information system to store and retrieve a variety of historical data on individual animals, including physical characteristics, geneology, sexual cycles, breeding history, reproductive history, and research and medical data. Many of these data categories can be quickly displayed via computer-generated tables or figures to summarize current or past colony status. The programs are interactive and designed to operate with a disk pack as the permanent storage unit for information on individual animals.

Restrictions. These programs employ virtual core arrays as the basic structure for each individual information file. When appropriate, sequential entry of information is accomplished by using two-dimensional arrays, where columns refer to specific information categories and rows contain the cumulative sequential entries. Virtual core storage requires considerable disk or core allocation, but is appropriate for an information system based on a colony size of 50 or fewer.

Pre-input. PRIME0 is a program to set up a virtual core file called "NAMES," which contains individual subfile headings and a one-dimensional array for the storage of individual animal identification designations (name or code). Current subfile headings include the following: (1) biographical data (identification code, cage location, birthdate, sex, type of rearing, sire, dam, full siblings, and half siblings; (2) biomedical data (sexual cycle, tuberculin tests, weight, and abnormal behavior; (3) breeding history (dates bred, duration of breeding session, mate identification, pregnancy test

Preparation of this report was supported by NICHD Grant 00973. date, pregnancy test result); (4) pregnancy data (infant birthdate, gestational age, type of birth, birth obseived, infant identification); (5) research history (experimental history, clinical-medical history, general comments). Of course, the specific subfiles can vary according to the user's needs.

Input. PRIME 1 is a program to create new files for individual animals or edit (add, change, or delete) information in existing files on an individual basis. When a new file is created, the user specifies the file name (usually the animal's identification) which is added to the NAMES file. Each subfile is contained in a single virtual core string matrix. The information category descriptive headings appropriate to each subfile are placed in the zero-th column of these arrays. Once the animal's file is opened, the user can specify individual subfiles to be accessed for initial input or editing. The biographical subfile is the only subfile not set up for sequential storage; all others store chronologically sequential entries along the rows of the subfile matrix. Individual text entries are limited in length according to information category. Entries in the biomedical data subfile are limited to 32 characters. Breeding and pregnancy subfiles are limited to 16 characters per entry.

Once the subfile is specified, the user is given the opportunity to make entries under each category in sequence. Dates are entered as six-digit numbers in the month/day/year format with the slashes located appropriately. A span of dates is entered as $\mathrm{MM} / \mathrm{DD}-\mathrm{DD} / \mathrm{YY}$, or MM/DD/YY-MM/DD/YY. The user is free to vary other text entries according to individual needs. For instance, under "pregnancy test result" can go not only a positive or negative designation, but also an abbreviation of the type of test used (i.e., abdominal palpation) and uterine length and width measures. Sexual cycle data is currently designed to record incidence of vaginal bleeding. Information from breeding programs where smears are taken, or perineal swellings are rated (Kuehn, Jensen, \& Morrill, 1965), can be substituted or added. Under "experimental history" a project number is used to indicate individual experiments in which the animal has served as subject. The remainder of the information categories are selfexplanatory.

Output. PRIME2 and PRIME3 are programs to print file contents either at the user's terminal or on a 132-character width line printer. PRIME2 prints tabled data. The user specifies any combination of subfiles or animals. Subfiles pertaining to data such as breeding history and pregnancy data are printed only for females. Also, the age of each animal to the nearest month at the time PRIME2 is run is printed.

PRIME3 performs the specialized function of plotting sexual cycle and breeding data for colony females. The current format for the plot is a 132-character width 
line printer page. Four consecutive months are located along the $X$ axis, with each print column equal to a single day. The $Y$ axis contains the identification code for each female animal. The recorded information includes an " $S$ " for days spotting, an " $M$ " for days of mating, a "+" or "-" for positive or negative pregnancy tests. The plotted figure displays this information for all colony females on a single page. The user can specify the particular 4-month block to be printed.

In general, the information contained in these virtual core arrays is readily transferable to a format suitable for statistical analysis.

Language and Computer. The programs are written in BASIC-PLUS for a DEC PDP $11 / 40$ computer, under the RSTS/E operating system.
Availability. Program listings and punched-tape versions are available at no charge from Richard Deni, Peabody College, Box 154, Nashville, Tennessee 37203.

\section{REFERENCES}

Digital Equipment Corporation. Basic-Plus language manual. Maynard, Mass: July 1975.

Kuehn, R. E., Jensen, G. D., \& MorRiLl, R. K. Breeding Macaca nemestrina: A program of birth engineering. Folia Primatologica, 1965, 3, 251-262.

National Research Council Committee on Conservation of Nonguman Primates. Nonhuman primates: Usage and availability for biomedical programs. Washington, D.C: National Academy of Sciences, 1975.

(Accepted for publication December 16, 1977.) 